\title{
La formación docente evaluada por método mixto
}

\author{
M. Esther Urrutia-Aguilar, Mónica B. Aburto-Arciniega, Angélica Arce-Cedeño, Rosalinda Guevara-Guzmán
}

Introducción. Actualmente, los planes de estudio de medicina en el mundo enfatizan el desarrollo de las competencias profesionales. Para ello es indispensable contar con programas de capacitación docente que incidan en el cambio de referentes pedagógicos de los profesores y adquirir así estrategias de enseñanza que se dirijan al logro del perfil del egresado. Para evaluar el cumplimiento de lo anterior, los beneficios de una metodología mixta son particularmente evidentes cuando se estudian iniciativas nuevas y sus interacciones en investigación educativa médica.

Objetivo. Evaluar por metodología mixta la formación docente de un grupo de profesores que asistieron a un curso-taller. Sujetos y métodos. Se evaluó cuantitativamente el impacto del curso por los cambios en los referentes pedagógicos de los profesores que asistieron al curso y cualitativamente por grupos focales que permitieron la agrupación de las narrativas.

Resultados. El referente tradicional después de la impartición del curso denotó una gran heterogeneidad entre tres de las estrategias (planeación, metodología y evaluación), no así con las de aprendizaje y relación docente-alumno; las estrategias en el referente alternativo y tecnología educativa se mantuvieron homogéneas. Los profesores expresaron que los cursos eran beneficiosos para la implementación de las competencias, pero que era necesario enfatizar más sobre la aplicación de las estrategias.

Conclusión. Los análisis cuantitativo y cualitativo mostraron que el curso-taller de formación docente coadyuvó a modificar la preferencia de los profesores por referentes pedagógicos distintos al tradicional, lo cual resulta de trascendencia para el desarrollo adecuado de un modelo educativo orientado a competencias.

Palabras clave. Estrategias de enseñanza. Evaluación mixta. Formación docente.

\section{Teaching training assessed through mixed-method}

Introduction. Medical curricula are currently focusing on the development of professional competences worldwide. In order to accomplish this, it is essential to have teaching training programs that influence on the pedagogical references change of teachers and develop teaching strategies that are addressed to the fulfillment of the graduate's profile. To achieve this, the benefits of mixed-method are particularly evident when new initiatives together with their interactions in medical education research are being studied.

Aim. To assess teaching training of a group of teachers attending a workshop through mixed-method.

Subjects and methods. The impact of the course was quantitatively assessed according to the pedagogical references changes and qualitatively through focal groups of teachers who had attended the workshop; this allowed the grouping of narratives.

Results. The conventional referent after taking the course showed a wide heterogeneity among three strategies (planning, methodology and assessment) opposite to those of learning and teacher-student relationship; the strategies in the alternative referent and teaching technology remained homogenous. Teachers reported that the courses were beneficial for implementing competences but that it was not necessary to emphasize the application of strategies.

Conclusion. The quantitative and qualitative analyses showed that the teaching training workshop contributed to the teachers' change in their pedagogical references preference other than the conventional one, which is completely determinant for the appropriate development of a teaching model oriented to competences.

Key words. Mixed-method. Teaching strategies. Teaching training.

Facultad de Medicina. Universidad Nacional Autónoma de México. México DF, México.

Correspondencia: Dra. María Esther Urutia Aguilar. Facultad de Medicina. Universidad Nacional Autónoma de México. Avda. Universidad, 3000. Circuito Interior. Ciudad Universitaria. CP 04510. México DF, México.

E-mail:

mariau@unam.mx

Financiación:

Apoyo financiero institucional PAPIME-DGAPA; número de registro PE401214 de la UNAM.

Agradecimientos: Profesora Josefina Bolado, por la revisión de estilo.

Conflicto de intereses: No declarado.

Competing interests: None declared.

(C) 2015 FEM 


\section{Introducción}

La Commission on Education of Health Professionals for the 21st Century, fundada en 2009 bajo una visión global que busca promover la salud, recomienda el desarrollo de innovaciones educativas e institucionales para formar una generación de profesionales en medicina que hagan frente a los problemas de sanidad presentes y futuros. Esta comisión enfatiza el desarrollo de competencias profesionales y reformas educativas [1].

En la Facultad de Medicina de la Universidad Nacional Autónoma de México (UNAM) se encuentra vigente el Plan de Estudios 2010, el cual se caracteriza por ser un currículo mixto por asignaturas con un enfoque por competencias [2]; fue aprobado por el Consejo Académico del Área de las Ciencias Biológicas, Químicas y de la Salud el 2 de febrero del 2010 e implementado en la generación 2011. En este plan de estudios, las competencias están descritas como 'la articulación y ponderación de conocimientos, habilidades, actitudes y valores que permiten tener un desempeño profesional eficiente con un juicio crítico y reflexivo en la práctica diaria en beneficio de los individuos y la sociedad'.

Los sistemas educativos dependen de la calidad y desempeño de sus docentes, de tal forma que constituyen una de las variables más significativas de la educación. Actualmente, no es suficiente para el profesor poseer un gran dominio de la materia que imparte, sino que a la vez debe contar con una actitud permanente de apertura al cambio y la capacidad de ir renovando la metodología de enseñanza. Dada la importancia de la labor docente, a partir de la implementación del Plan de Estudios 2010 de la Facultad de Medicina, se justifica la necesidad de sensibilizar a los profesores para integrarlos a un modelo educativo orientado al desarrollo de competencias. Por lo anterior, resulta indispensable contar con programas de capacitación docente que incidan en el cambio de referentes pedagógicos de los profesores y adquirir estrategias de enseñanza que sean dirigidas al logro del perfil del egresado.

Las estrategias de enseñanza son definidas como los procedimientos que el profesor utiliza de manera flexible, adaptativa, autorregulada y reflexiva para promover el aprendizaje significativo en los estudiantes [3], sustentadas en el concepto particular del profesor sobre cómo aprenden los alumnos.

Estas estrategias de enseñanza se pueden agrupar en sustentos teóricos que permitan conformar los referentes pedagógicos que, según Martínez et al
[4], se clasifican en: tradicional, tecnología educativa y alternativo.

En un estudio anterior se describió que los profesores sí tuvieron cambios en los referentes, sobre todo en la concepción del aprendizaje, donde la tendencia a utilizar el referente tradicional disminuyó. Sin embargo, en la relación alumno-profesor predominó el referente tradicional [5], por lo cual el cursotaller que se considera en este estudio se reestructuró reforzando los temas que abordan dicha temática.

Actualmente, en el ámbito de la investigación educativa médica se utilizan los métodos mixtos con la finalidad de ampliar el análisis de un determinado objetivo a investigar, ya que lo cuantitativo arroja evidencias de respuestas que pueden ser generalizables y lo cualitativo profundiza a partir de descripciones personales del fenómeno en estudio [6-8]. Los beneficios de una metodología mixta son particularmente evidentes cuando se estudian iniciativas nuevas y sus interacciones en investigación educativa médica [9]. Por estas bondades, el objetivo de este trabajo es evaluar la formación docente de un grupo de profesores que asistieron a un curso-taller a través de una metodología mixta.

\section{Sujetos y métodos}

Se realizó un estudio mixto de tipo cuantitativo y cualitativo [9], observacional, descriptivo y longitudinal. La población de estudio fue de 17 ayudantes de profesor (el 55\% del total de su categoría) que participaron de manera voluntaria en el curso-taller; además, representan la tercera parte de la plantilla docente de un departamento académico del área morfológica. Los ayudantes de profesor, que se encuentran cursando la licenciatura de médico cirujano, corresponden a los docentes con menor experiencia y son seleccionados a través de un proceso académico por un cuerpo colegiado. Su práctica docente la llevan a cabo bajo la supervisión y guía del profesor titular.

Para la obtención de datos cuantitativos se aplicó una evaluación pretest-postest a través de un instrumento sobre el concepto de enseñanza relacionado con tres referentes pedagógicos (tradicional, tecnología educativa y alternativo). El instrumento está conformado por 42 ítems, con escala tipo Likert, agrupados en cinco estrategias:

- Aprendizaje (7 items): indaga sobre las creencias que tiene el profesor acerca de la responsabilidad de aprender y enseñar.

- Planeación (4 ítems): se refiere a la organización de las actividades en torno a la enseñanza. 
- Metodología (17 ítems): empleada por el profesor según su creencia personal de cómo abordar los contenidos del programa académico; por ejemplo, tomar en cuenta las habilidades del estudiante.

- Evaluación (5 items): explora el propósito que ésta tiene para el profesor (cumplir con los objetivos, realimentación, entre otros).

- Relación docente-estudiante (9 items): investiga sobre las creencias del profesor con respecto a la relación que establece con sus estudiantes.

Este instrumento cuenta con validez de contenido y fiabilidad descritos en la bibliografía ( $\alpha$ de Cronbach: $0,77)[4]$.

Para agrupar los resultados, se obtuvieron frecuencias y porcentajes de las respuestas de cada uno de los ítems según acuerdo o desacuerdo, se clasificaron conforme al referente pedagógico (tradicionales, tecnología educativa y alternativos) al que pertenecían y se procedió a la agrupación por estrategia (aprendizaje, planeación, metodología, evaluación y relación docente-estudiante).

La evaluación cualitativa se llevó a cabo por la técnica de grupo focal, en la cual se busca estimular la conversación relacionada con el impacto del 'Curso-taller de formación docente en competencias', con el objetivo de provocar narrativas explicativas que den cuenta de la experiencia vivida en el proceso de aprendizaje durante la intervención del curso-taller [10]. Se desarrolló una guía de entrevista semiestructurada, con base en los temas abordados en el curso-taller, que constó de 11 preguntas abiertas (Tabla I).

El grupo focal se llevó a cabo al término del curso-taller y fue conducido por uno de los investigadores de este proyecto. La entrevista se grabó y transcribió de forma literal; posteriormente, se elaboró un árbol de categorías a partir de la guía y las respuestas obtenidas por los participantes y los resultados se analizaron según el uso de análisis temático [11] sobre los ítems de competencias, aprendizaje autorregulado y colaborativo, aplicación de las estrategias de enseñanza, planeación y evaluación.

El curso-taller se realizó en los meses de enero a marzo de 2014, con una duración de 30 horas. El diseño instruccional del curso reflejó una aproximación constructivista. En las sesiones se promovió la participación activa de los integrantes y la divergencia de opiniones respecto a cada uno de los temas.

Los temas abordados versaron sobre constructivismo, aprendizaje centrado en el alumno, aprendizaje colaborativo, aprendizaje autorregulado, manejo del aula de clases, ambientes de aprendizaje,
Tabla I. Guía de entrevista estructurada de grupos focales.

¿Considera que las estrategias enseñadas en el curso le permitirán desarrollar las competencias en los estudiantes?

¿Considera que los temas abordados fueron los indicados para enseñarle cómo planear una clase?

¿El curso le ayudó a determinar qué tipo de estrategias constructivistas implementar y cuáles fueron?

¿El curso promovió la enseñanza para elaborar razonamiento clínico en los estudiantes?

¿En el curso se promovió la importancia de la reflexión?

¿Se promovió la comunicación para apoyar la enseñanza y para establecer comunicación entre profesores y estudiantes? ¿Cómo?

Después del curso, ¿se siente más seguro de las estrategias de enseñanza que conoce en comparación con las que contaba antes del curso? ¿Por qué?

¿Considera que el curso le ayudó a modificar sus referentes pedagógicos? ¿Cómo los modificó?

¿Cuáles fueron los beneficios de asistir al curso?

¿Qué fue lo más positivo y lo más negativo del curso?

¿Podría hacer una propuesta para mejorar el curso?

manejo del conflicto, métodos de enseñanza y evaluación de la enseñanza por competencias.

Se aplicó estadística descriptiva y la diferencia entre las proporciones del referente pedagógico tradicional, antes y después del curso, se analizó mediante la prueba chi al cuadrado de hipótesis de McNemar. El programa estadístico usado fue SPSS v. 20.0.

\section{Resultados}

En total, participaron 17 profesores que respondieron las pruebas pretest-postest. En promedio, estos profesores han desempeñado labores docentes durante dos años (rango: 2-4 años).

En la tabla II se presentan los resultados por referente considerando el porcentaje de respuestas de acuerdo a lo que los profesores contestaron antes y después de asistir al curso. Se denota que el curso impactó con diferencias significativas $(p \leq 0,05)$ en el referente tradicional en las estrategias de aprendizaje, metodología y relación docente-alumno. Los referentes de conceptualización alternativa y de tecnología educativa no tuvieron cambios significativos, con excepción de la relación docente-alumno.

En la figura, los círculos muestran la relación entre las cinco estrategias de los tres referentes peda- 
Tabla II. Porcentaje de acuerdo de los profesores, previo y posterior al curso, según referente pedagógico.

\begin{tabular}{|c|c|c|c|c|c|c|c|}
\hline & \multicolumn{3}{|c|}{ Tradicional } & \multicolumn{2}{|c|}{ Alternativa } & \multicolumn{2}{|c|}{ Tecnología educativa } \\
\hline & Previo (\%) & Posterior (\%) & $\chi^{2}$ & Previo (\%) & Posterior (\%) & Previo (\%) & Posterior (\%) \\
\hline Aprendizaje & 83 & 70 & 0,05 & 97 & 100 & 93 & 100 \\
\hline Planeación & 19 & 12 & NS & 88 & 94 & 97 & 91 \\
\hline Metodología & 61 & 53 & 0,05 & 92 & 96 & 78 & 75 \\
\hline Evaluación & 37 & 37 & NS & 63 & 91 & 91 & 94 \\
\hline Relación docente-alumno & 94 & 69 & 0,05 & 69 & 79 & & \\
\hline
\end{tabular}

$\chi^{2}$ de hipótesis de McNemar. NS: no significativo.

gógicos al término del curso-taller. Con respecto al referente tradicional después de la impartición del curso, se denota una gran heterogeneidad entre tres de las estrategias (planeación, metodología y evaluación), no así en las de aprendizaje y relación docentealumno; las estrategias en el referente alternativo y tecnología educativa se mantuvieron homogéneas.

Al realizar el análisis del porcentaje de profesores con y sin cambios en el referente tradicional desglosando cada estrategia en el pretest y postest (Tabla III), se puede observar que el mayor porcentaje sin cambios fue en evaluación $(87,5 \%$ ) y en planeación $(81,25 \%)$, ya que desde el pretest, el profesor expresó estar más de acuerdo con el referente de tecnología educativa.

\section{Grupos focales}

De los profesores con un mínimo de $80 \%$ de asistencia al curso-taller, se seleccionaron de manera aleatoria nueve posibles participantes para el grupo focal (seis mujeres y tres hombres). Previa explicación verbal del objetivo del estudio, los nueve profesores aceptaron participar de manera voluntaria.

En el tema de competencias, los participantes indicaron que el curso-taller les ayudó a entender la aplicación de las estrategias de enseñanza para lograr el desarrollo de las competencias en los estudiantes: 'Si bien es cierto que algunas de las estrategias ya las conocía, la forma de aplicarlas con los alumnos, realmente, no tanto. Las dinámicas que vimos de cómo aplicar esas estrategias de aprendizaje en el desarrollo de las competencias, para mí son innovadoras porque yo no lo había hecho'.

También comentaron que faltaban ejercicios que les brindaran más claridad en su aplicación: 'Siento que faltó centrarse un poco más en cada una de las competencias; de todas las estrategias que vimos, yo desconocía muchas de ellas. Sin embargo, algunas no conseguí asimilarlas, no logré ver dónde podrían aplicarse directamente o en algunas ni siquiera pude conocer realmente de qué se trataba la estrategia'.

En el tema de autorregulación y aprendizaje colaborativo opinaron que sí les fue de ayuda para poder orientar a los estudiantes, y que estrategias como el aprendizaje basado en problemas les proporcionaron conocimientos para su aplicación: 'Creo que el hecho de enseñar a los alumnos las diferentes formas en las que pueden manejar su aprendizaje, ya sea con cuadros, mapas mentales, resúmenes o lo que a ellos les sea más útil, ayuda a que se promueva el aprendizaje autorregulado'; 'Lo que hace el aprendizaje basado en problemas es empoderarte de conocimiento, hacerte responsable de lo que vas a buscar; creo que favorece muchísimo las competencias y, de hecho, es la que integra más comunicación y profesionalismo. Tienes que integrar todo, es donde en realidad se genera más búsqueda de información y el alumno se queda con ganas de seguir buscando más y compartir con los compañeros su información'.

En la aplicación de las estrategias de aprendizaje, algunos profesores compartieron que el curso-taller les fue de gran ayuda en el conocimiento de la diversidad de estilos de aprendizaje de los estudiantes: 'Creo que este curso sí aportó algo porque cuando estás hablando a los alumnos de estrategias de aprendizaje, das a entender que te interesas por ellos; te ayudan a conocerlos mejor, y si conoces mejor a tus alumnos, entonces ellos te tienen más confianza y puedes ejercer una interacción mucho más directa. Se sienten con la libertad de expresarse, de pregun- 
tar sus dudas y otras cuestiones que no hayan entendido y de interaccionar con sus compañeros'.

Otros consideraron que en este curso faltó conocer con mayor amplitud la aplicación de las estrategias: 'Fue evidente que las estrategias se dieron a conocer; lo negativo es que quizá fue poco tiempo para toda la información y que faltó enfocar más las actividades en base a las estrategias que se iban dando.'

Sobre los temas de planeación realizados en el curso-taller, los docentes consideraron que fueron de gran utilidad y en los que más reflexionaron: 'Te ayuda a organizar un poco más la actividad y a tener planeada la clase desde el inicio. A mí me ayudó a poder idear y a ver la importancia de conocer bien a tus alumnos, saber quiénes son, cómo son, para en base a ello determinar qué estrategia utilizar'; 'quizá yo era más visual y consideraba que para los alumnos era más fácil aprender así, pero el curso me ayudó a darme cuenta de que la gente aprende de diferente manera y a considerar que es todo un reto poder planear estrategias'.

En el tema de evaluación, los participantes en el curso comentaron que sí fue de utilidad, aunque les parece un tema complejo y que debería existir un curso sólo con esta temática: 'A mí me sirvió mucho, ya que conocí más formas de poder evaluar a los alumnos. Realmente estaba muy ligada a la idea de que evaluar eran sólo exámenes y participaciones en clase; sin embargo, la forma de evaluar es algo más dinámico, no sólo cuenta el conocimiento, sino que también estamos hablando de la actitud de cada alumno, de su interés, de la forma en la que va construyendo sus conocimientos'; 'conocer las listas de cotejo me pareció una manera no muy objetiva; creo que hay que enfatizar un poco más la utilización de los instrumentos, y que faltó tiempo para centrarse más en este tema, a lo mejor se precisa un curso exclusivo de este tema'.

Sobre las mejoras en el curso, todos los participantes coincidieron en que debería implementarse más práctica: 'Faltó un poco la práctica de las estrategias. Creo que estaría bien que en los cursos posteriores tratara de implementarse, aunque sé que es un poco complicado por el tiempo. La información era muy buena porque la calidad de información que se manejó en el curso lo era, pero lo negativo era que faltaba aplicarlo'.

\section{Discusión}

Los resultados indican que el curso-taller de formación docente diseñado con una aproximación constructivista influyó en los profesores en sus referen-
Figura. Porcentaje de acuerdo de los profesores con los referentes pedagógicos y estrategias de enseñanza en el pretest-postest.

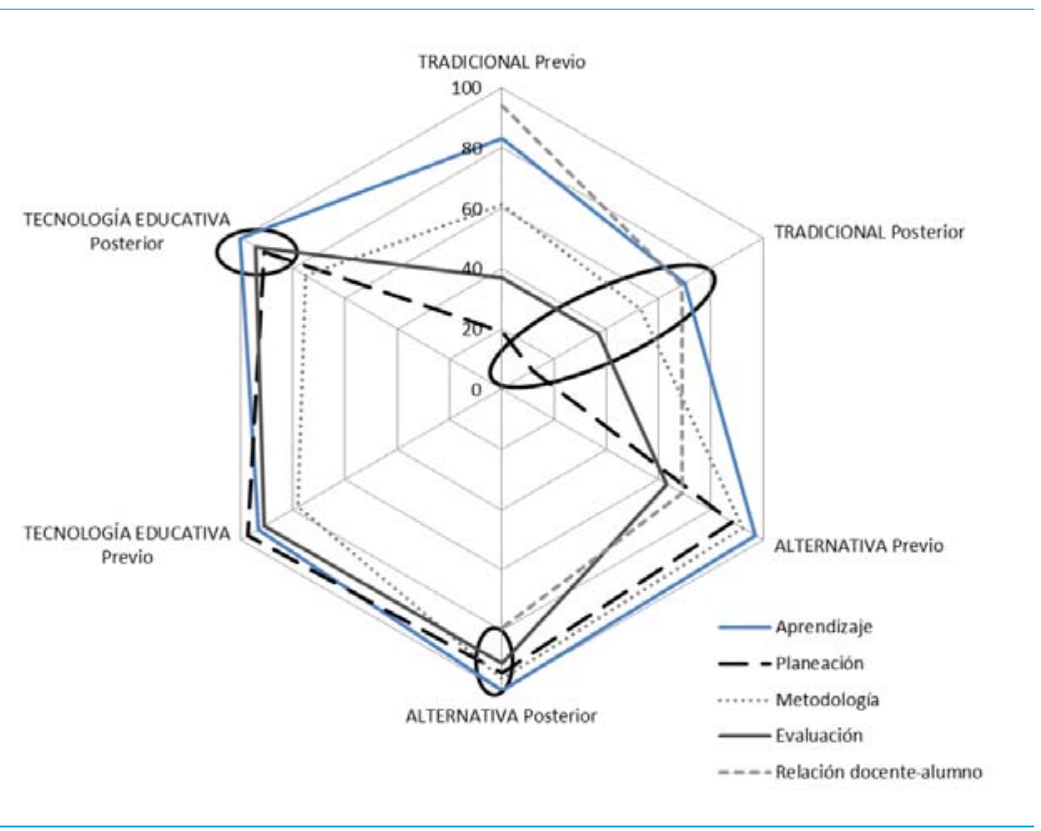

Tabla III. Porcentaje de profesores con y sin cambios según el pretest y postest en el referente tradicional, desglosando cada estrategia.

\begin{tabular}{lccc}
\hline & Acuerdo-desacuerdo & Desacuerdo-acuerdo & Sin cambios \\
\hline Aprendizaje & 25 & 12,5 & 62,5 \\
\hline Planeación & 12,5 & 6,25 & 81,25 \\
\hline Metodología & 62,5 & 25 & 12,5 \\
\hline Evaluación & 6,25 & 6,25 & 87,5 \\
\hline Relación docente-alumno & 6,25 & 93,75 & 0 \\
\hline
\end{tabular}

tes pedagógicos, de manera directa en el tradicional; muestran también que la reflexión a partir de lecturas, ejercicios y vivencias grupales incidió en los pensamientos o en las creencias que se tenían sobre las estrategias de enseñanza.

Sin embargo, hay que destacar la gran heterogeneidad existente antes y después del curso entre los referentes pedagógicos y las estrategias de enseñanza; con relación al aprendizaje, los profesores están de acuerdo en que éste depende más de los conocimientos que el profesor proporcione y de la memorización de los estudiantes (tradicional), pero en la estrategia de planeación están más de acuerdo en 
que debe llevarse a cabo con base en objetivos que propicien la participación de los alumnos (tecnología educativa); en cuanto a la estrategia evaluativa, están de acuerdo en promover la autoevaluación y la realimentación (alternativo). Lo anterior denota la falta de formación pedagógica, y aunque en algunos profesores se refleja un cambio después del curso, no fue suficiente para lograr la cohesión de todas las estrategias. Algunas investigaciones sostienen que el acercamiento a la enseñanza depende del campo disciplinar de donde provenga el profesor; es decir, las formas de pensar según cada disciplina acaban por ejercer una fuerte influencia en la práctica docente. Lueddeke [12] y Trigwell y Prosser [13], en dos estudios diferentes, comprobaron que los profesores universitarios de las consideradas ciencias 'duras', como la física, la ingeniería y la medicina, sostenían un acercamiento centrado en la materia, mientras que los profesores de ciencias sociales y humanidades manifestaban un acercamiento centrado en el alumno [14].

Con relación a la heterogeneidad de las estrategias de enseñanza (planeación, metodología y evaluación) en el referente tradicional después del curso, se debe a que los efectos de la formación pedagógica en las concepciones y creencias sobre la enseñanza no se producen de manera inmediata -sólo por el hecho de haber participado en un curso de formación-, sino que requieren la instauración de capacidades reflexivas en y sobre la propia enseñanza y la adquisición de habilidades que conduzcan al profesor a estar continuamente interpretando y analizando la docencia y la realidad científica a la que pertenece [15].

Además, la población de estudio estuvo constituida por profesores jóvenes, con alguna experiencia profesional y con menos de tres años de impartir docencia en una institución universitaria. Por tanto, lo expresado en el grupo focal coincide con lo comunicado en la bibliografía: por ejemplo, pensar que enseñar en la universidad es un reto (a veces demasiado grande) y que han tenido experiencias como estudiantes con profesores que les parecían aburridos y no quieren parecerse a ellos, por lo que están dispuestos a hacer lo que haga falta para ser buenos docentes [16].

Pensar o querer probar que el curso generó grandes procesos de cambio sería una atribución errónea, ya que el cambio en los referentes pedagógicos obedece a diferentes fuentes. Más bien, los resultados fueron beneficiosos para los profesores al permitirles una reflexión de los aprendizajes, dado que en el grupo focal reconocieron el sentido y significado que tuvo la participación en un programa de formación pedagógica que les ayudó a comprender la docencia desde su propia realidad, a entender la diversidad en el aprendizaje de los alumnos y, en base a ello, planear la didáctica. Por esto, una de las direcciones futuras de este proyecto es llevar a cabo una evaluación posterior para dar seguimiento de este posible cambio en los referentes pedagógicos y creencias docentes. Como referencia, Herskovic et al [17] describieron los cambios en la percepción de un grupo de profesores respecto de su práctica docente a un año de haber participado en tres cursos de formación orientados al currículo, metodología y evaluación por competencias, resaltando su preferencia por enfocar mayormente su práctica docente al logro de competencias; esto refleja la bondad de este tipo de estrategias. Así, puede resultar pertinente hacer un análisis similar con los profesores que participaron en este estudio y determinar el impacto de los cursos impartidos, a largo plazo, sobre el quehacer docente.

El curso también permitió revalorar el sentido de la evaluación, ya que los profesores manifestaron en el grupo focal la importancia de la evaluación formativa y de conocer diferentes instrumentos que se pueden utilizar para evaluar a los alumnos.

La principal limitación de este trabajo fue la muestra reducida y que estaba constituida por profesores jóvenes con poca experiencia docente, por lo que no pueden generalizarse los resultados a toda la plantilla docente.

En conclusión, los resultados de este estudio, según el análisis cuantitativo y cualitativo, mostraron que el curso-taller de formación docente influyó en modificar la preferencia de los docentes por referentes pedagógicos distintos al tradicional, lo cual resulta de trascendencia para el desarrollo adecuado de un modelo educativo orientado a competencias. Fue evidente la dispersión conceptual y teórica existente en el ámbito de pensamientos y creencias del profesor, que es similar a lo comunicado en otros estudios, donde han manifestado que en los docentes existe una falta de coherencia epistemológica entre los elementos de los modelos didácticos [18]. Desde esta perspectiva, los cambios en las concepciones y en la práctica docente se conciben lentos y graduales, con importantes resistencias y dificultades [19]. A pesar de ello, las creencias de los profesores no son independientes entre ellas, sino que tienen una estructura interna de carácter psicológico, no necesariamente lógico, y no todas las creencias son igualmente importantes para el individuo o son igualmente resistentes al cambio [20].

En este trabajo se constata que la metodología mixta es un referente valioso para confirmar que no 
sólo lo medido tiene valor (resultados cuantitativos), sino también los pensamientos por medio de las narraciones, ya que permiten entender lo que ocurre sin excluir la participación de las emociones y sentimientos [21].

El reto es considerar que, en la formación docente, la distancia entre conocimientos declarativos (conocimiento teórico) y saber práctico (conocimientos procedimentales) sigue siendo una brecha grande que habrá que reducir incorporando nuevas modalidades de formación basadas en la concepciones de la praxis. Además, los contenidos de estos cursos deben estructurarse según las necesidades de los docentes para que éstos adquieran competencias y destrezas, conocimientos y actitudes adecuados para llevar a cabo una enseñanza de calidad.

\section{Bibliografía}

1. Bhutta ZA, Chen L, Cohen J, Crisp N, Evans T, Fineberg H, et al. Education of Health Professionals for the 21st Century: a global independent commission. Lancet 2010; 375: 1137-8

2. Sánchez-Mendiola M, Durante-Montiel I, Morales-López S, Lozano-Sánchez R, Martínez-González A, Graue-Wiechers E. Plan de Estudios 2010 de la Facultad de Medicina de la Universidad Nacional Autónoma de México. Gac Med Mex 2011; 147: 152-8.

3. Díaz-Barriga F, Hernández-Rojas G. Estrategias docentes para un aprendizaje significativo. México DF: McGraw-Hill Interamericana; 2010

4. Martínez GA, Ferrés GA, Gutiérrez DS. Referentes pedagógicos y estrategias de enseñanza de los profesores de la Maestría en Ciencia Bioquímicas de la UNAM. Rev Anuies 2001; 4: 54-9.

5. Urrutia AM, Guevara GR, Aburto AM. Efecto de un cursotaller de formación docente sobre los referentes pedagógicos de profesores de embriología. Rev Inv Educ Med 2014; 3: 232.

6. Creswell JW, Plano CV. Designing and conducting mixed methods research. Thousand Oaks, CA: Sage Publications; 2007.
7. Stange KC, Zyzanski SJ. Integrating qualitative and quantitative research methods. Fam Med 1989; 21: 448-51.

8. Tashakkori A, Teddlie C. Mixed methodology: combining qualitative and quantitative approaches. Thousand Oaks, CA: Sage Publications; 1998.

9. Schifferdecker KE, Reed VA. Using mixed-methods research in medical education: basic guidelines for researchers. Med Educ 2009; 43: 637-44.

10. Mays N, Pope C. Rigor and qualitative research. BMJ 1995; 311: 109-12.

11. Braun V, Clarke V. Using thematic analysis in psychology. Qual Res Psychol 2006; 3: 77-101.

12. Lueddeke G. Professionalising teaching practice in higher education: a study of disciplinary variation and 'teaching scholarship'. Studies in Higher Education 2003; 28: 213-28.

13. Trigwell K, Prosser M. Congruence between intention and strategy in university science teachers' approaches to teaching. Higher Education 1996; 32: 77-87.

14. Lindblom YS, Trigwell K, Nevgi A, Ashwin P. How approaches to teaching are affected by discipline and teaching context. Studies in Higher Education 2006; 3: 285-98.

15. Postareff L, Lindblom YS, Nevgi A. A follow-up study of the effect of pedagogical training on teaching in higher education. Higher Education 2008; 56: 29-43.

16. Gibbs GP, Habeshaw T. Preparing to teach: an introduction to effective teaching in higher education. Bristol: Technical and Educational Service; 1992.

17. Herskovic P, Miranda T, Cortés E, Delucchi A, Gómez P, Jiusán $\mathrm{A}$, et al. ¿Creen haber cambiado los docentes un año después de un curso de docencia clínica? Educ Med 2012; 15: $179-84$

18. Porlán R, Martín del Pozo R. ¿Cómo progresa el profesorado al investigar problemas prácticos relacionados con la enseñanza de la ciencia? Revista Alambique 2006; 48: 92-9.

19. Mellado V. Cambio didáctico del profesorado de ciencias experimentales y filosofía de las ciencias. Enseñanza de las Ciencias 2003; 21: 343-58.

20. Bryan LA. Research on science teacher beliefs. In Fraser BJ, Tobin K, McRobbie CJ, eds. Second international handbook of science education. Dordrecht: Springer; 2012. p. 477-95.

21. Lifshitz A, Halabe J, Ramiro M. El valor de lo subjetivo. Gac Med Mex 2013; 149: 485. 